\title{
Relative abundance and structure of chaotic behavior: The nonpolynomial Belousov- Zhabotinsky reaction kinetics
}

Cite as: J. Chem. Phys. 131, 044105 (2009); https://doi.org/10.1063/1.3168400

Submitted: 08 April 2009. Accepted: 12 June 2009 . Published Online: 22 July 2009

Joana G. Freire, Richard J. Field, and Jason A. C. Gallas

\section{ARTICLES YOU MAY BE INTERESTED IN}

Deterministic chaos in the Belousov-Zhabotinsky reaction: Experiments and simulations

Chaos: An Interdisciplinary Journal of Nonlinear Science 3, 723 (1993); https://

doi.org/10.1063/1.165933

Oscillations in chemical systems. IV. Limit cycle behavior in a model of a real chemical reaction The Journal of Chemical Physics 60, 1877 (1974); https://doi.org/10.1063/1.1681288

"Black spots" in a surfactant-rich Belousov-Zhabotinsky reaction dispersed in a water-in-oil microemulsion system

The Journal of Chemical Physics 122, 174706 (2005); https://doi.org/10.1063/1.1888386

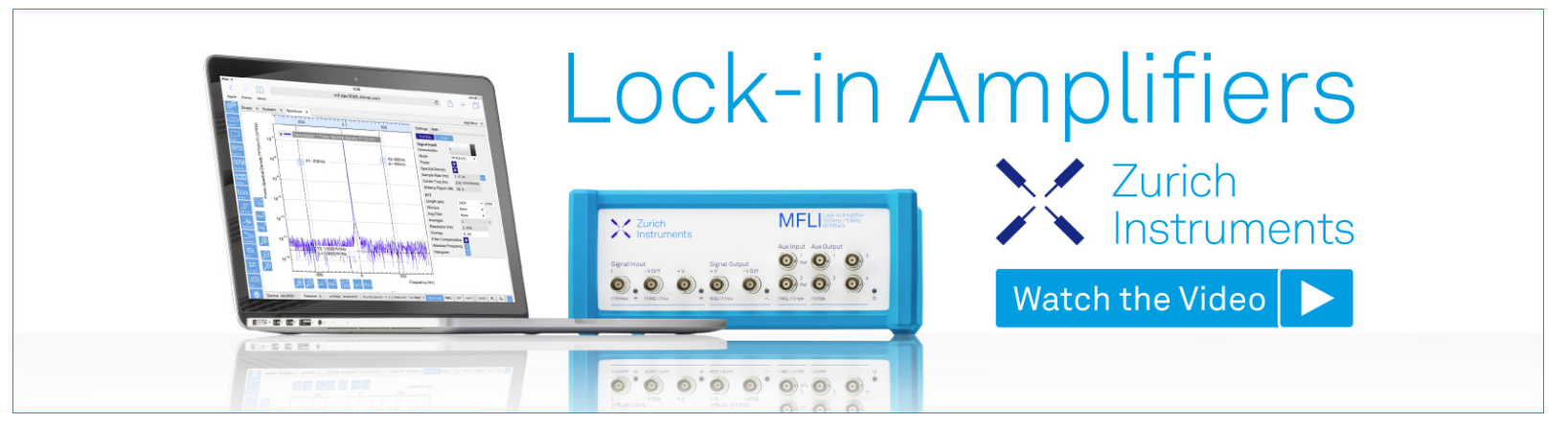

J. Chem. Phys. 131, 044105 (2009); https://doi.org/10.1063/1.3168400 


\title{
Relative abundance and structure of chaotic behavior: The nonpolynomial Belousov-Zhabotinsky reaction kinetics
}

\author{
Joana G. Freire, ${ }^{1,2}$ Richard J. Field, ${ }^{3}$ and Jason A. C. Gallas ${ }^{1,4, a)}$ \\ ${ }^{1}$ Instituto de Física, Universidade Federal do Rio Grande do Sul, 91501-970 Porto Alegre, Brazil \\ ${ }^{2}$ Centro de Estruturas Lineares e Combinatórias, Universidade de Lisboa, 1649-003 Lisboa, Portugal \\ ${ }^{3}$ Department of Chemistry, The University of Montana Missoula, Montana 59812, USA \\ ${ }^{4}$ Rechnergestützte Physik der Werkstoffe IfB, ETH Hönggerberg, HIF E12, CH-8093 Zurich, Switzerland
}

(Received 8 April 2009; accepted 12 June 2009; published online 22 July 2009)

\begin{abstract}
We report a detailed numerical investigation of the relative abundance of periodic and chaotic oscillations in phase diagrams for the Belousov-Zhabotinsky (BZ) reaction as described by a nonpolynomial, autonomous, three-variable model suggested by Györgyi and Field [Nature (London) 355, 808 (1992)]. The model contains 14 parameters that may be tuned to produce rich dynamical scenarios. By computing the Lyapunov spectra, we find the structuring of periodic and chaotic phases of the BZ reaction to display unusual global patterns, very distinct from those recently found for gas and semiconductor lasers, for electric circuits, and for a few other familiar nonlinear oscillators. The unusual patterns found for the BZ reaction are surprisingly robust and independent of the parameter explored. (C) 2009 American Institute of Physics.
\end{abstract}

[DOI: $10.1063 / 1.3168400]$

\section{INTRODUCTION}

It is well known that several chemical reactions are capable of displaying both periodic and chaotic oscillations in the concentrations of reactive intermediate species. The Belousov-Zhabotinsky reaction (BZR) is a paradigm of this rich dynamical behavior, investigated in many papers and featured in several books. ${ }^{1-4}$ However, the quantification of the relative abundance and structural distribution of chaos and periodicity in parameter space of this system has remained poorly investigated. Rather than reflecting a lack of interest, this spotty knowledge reflects the large computational effort required to construct phase diagrams for phenomena represented by flows in phase space, i.e., by continuous-time dynamical systems governed by sets of differential equations. While it is easy to iterate discrete maps, it is far harder and time consuming to integrate differential equations. For this reason, most of the accumulated knowledge about chaotic behavior in natural phenomena, phase diagrams in particular, comes from investigations based on discrete-time nonlinear maps.

Exploration of the parameter space of dynamical systems governed by differential equations has attracted some attention recently, following a report ${ }^{5}$ that the phase diagram of a loss-modulated $\mathrm{CO}_{2}$ laser, a flow, is surprisingly similar to that of a textbook example of a discrete-time dynamical system, the Hénon map. The immediate question is what other sort of vector flows might produce isomorphically similar phase diagrams and what new features they might have. Surprising bifurcation phenomena have been reported recently for systems across distinct disciplines and with various motivations. ${ }^{5-15}$ For a survey, see Ref. 16.

Generically, the most obvious regularities observed so

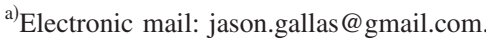

far in phase diagrams of flows consist of sequences of selfsimilar periodicity islands spread in chaotic phases, "shrimps," underly period-doubling cascades. The rich bifurcation phenomena found in flows so far contain novel features that emerge organized in regular patterns not known in discretetime systems (maps). This novelty is related to the distinct manners that shrimps are "glued" together to form regular patterns over extended regions in parameter space. However, the basic organizational Leitmotiv is still based on shrimp networks that accumulate systematically in one way or another. ${ }^{6,12}$ Thus, is it possible to find macroscopic regularities of a different kind, i.e., other than glued shrimps?

A common feature of the models already investigated is that they are mainly governed by polynomial equations of motion. So, what can one expect from systems governed by nonpolynomial equations of motion? We find novel and unexpected features in a nonpolynomial system and describe them in some detail.

Although nonpolynomial models of dynamical systems exist abundantly in literature, their chaotic phases have been explored mainly by plotting bifurcation diagrams along a few specific one-parameter cuts. Moreover, phase diagrams normally reported do not describe details of the chaotic phases ${ }^{19}$ but, instead, focus mainly on bifurcation boundaries between regions involving periodic oscillations of small periods. We investigate here the relative abundance and structuring of the chaotic phases for a nonpolynomial model involving three independent variables and 14 parameters and describing BZR chaos. ${ }^{20}$ This model is complex in the sense of Nazarea and Rice. ${ }^{21}$ Bifurcation diagrams for this model display features resembling those recently found near certain hubs in phase diagrams. ${ }^{1,16}$ Because not many hubs are presently known, and there is no theoretical method to anticipate the location of hubs, we perform a detailed numeri- 
cal investigation of the model. Although hubs were not found, we do find a rather unusual structuring (described below): fountainlike global patterns consisting of alternate eruptions of chaos and periodicities. Models of real chemical reactions are particularly appealing in that they arise from experiment and, therefore, dynamical behaviors predicted from them should be amenable to experimental verification.

\section{THE BZ REACTION}

The classic $\mathrm{BZR}^{2,22,23}$ is the cerium-ion $\{\mathrm{Ce}(\mathrm{IV}) / \mathrm{Ce}(\mathrm{III})\}$ catalyzed oxidation of malonic acid $\left\{\mathrm{CH}_{2}(\mathrm{COOH})_{2}\right\}$ by bromate ion $\left\{\mathrm{BrO}_{3}^{-}\right\}$in aqueous sulfuric acid $\left\{\mathrm{H}_{2} \mathrm{SO}_{4}\right\}$ media. ${ }^{24}$ In a well-stirred, closed reactor, damped temporal oscillations occur in the concentrations of various intermediate species, e.g., $\mathrm{Ce}(\mathrm{IV}), \mathrm{Ce}(\mathrm{III}), \mathrm{BrO}_{2}, \mathrm{HBrO}_{2}, \mathrm{HOBr}$, and $\mathrm{Br}^{-}$. The concentration of bromalonic acid (BrMA) is an important dynamic quantity that serves as a bifurcation parameter for the onset and eventual disappearance of oscillation in a closed reactor.

True chemical steady states may be achieved when the BZR is run in a continuous flow, stirred tank reactor (CSTR), into which solutions containing the reactants are pumped while reaction mixture overflows. ${ }^{2,3}$ This makes the critical bifurcation species BrMA (vide infra) a dynamic, oscillatory variable. Oscillatory or even chaotic ${ }^{4}$ stationary states often appear.

Deterministic chaos was first observed in BZ-CSTR experiments in 1977 by Schmitz et al. ${ }^{25}$ and better characterized by Hudson and Mankin. ${ }^{26}$ The appearance of periodicchaotic windows as the flow rate was monotonically increased was soon observed by Turner et $a l^{27}$ and by Vidal et al. $^{28}$ Several classic transitions from periodicity to chaos were observed in 1983 by Roux. ${ }^{29}$ The observed chaotic states generally run from mixed-mode systems at low flow rates $^{27,28}$ to more complex behaviors at higher flow rates. ${ }^{30}$

There is some uncertainty concerning the origin of BZCSTR chaos. Low-flow-rate chaos can be well reproduced ${ }^{31}$ by a model based only on the homogeneous chemistry ${ }^{24}$ of the BZR. However, there is considerable experimental evidence that high-flow-rate BZ-CSTR chaos may be at least strongly affected by imperfect mixing effects. ${ }^{32-35}$ The model under consideration here is based only on BZR chemistry.

The basic chemistry of the BZR is referred to as the Field, Körös, Noyes ${ }^{24}$ (FKN) mechanism. The FKN mechanism may be reduced to a skeleton form referred to as the Oregonator ${ }^{36,37}$ involving only the species $\mathrm{HBrO}_{2}, \mathrm{Br}^{-}$, and $\mathrm{Ce}(\mathrm{IV})$. However, while both the FKN mechanism and the Oregonator at least qualitatively reproduce in simulations the BZR oscillations, neither model has been found to generate chaos. Györgyi and Field ${ }^{38}$ expanded the details of the reactions of BrMA and the malonyl radical to produce a threevariable model ${ }^{20}$ that reproduces well the periodic-chaotic nature of the low-flow-rate chaos. This model is based upon the nonstoichiometric chemical reactions (1)-(7) and leads to a set of three nonpolynomial differential equations. The left hand sides of Eqs. (1)-(7) are rate determining for the appearance of products on the right hand sides,

$$
\begin{aligned}
& \mathrm{Br}^{-}+\mathrm{HBrO}_{2}+\mathrm{H}^{+} \rightarrow 2 \mathrm{krCH}(\mathrm{COOH})_{2}, \\
& \mathrm{Br}^{-}+\mathrm{BrO}_{3}^{-}+2 \mathrm{H}^{+} \stackrel{k_{2}}{\rightarrow} \mathrm{BrCH}(\mathrm{COOH})_{2}+\mathrm{HBrO}_{2},
\end{aligned}
$$

$$
\begin{aligned}
& 2 \mathrm{HBrO}_{2} \rightarrow \mathrm{BrCH}(\mathrm{COOH})_{2}, \\
& \frac{1}{2} \mathrm{HBrO}_{2}+\mathrm{BrO}_{3}^{-}+\mathrm{H}^{+} \stackrel{k_{4}}{\rightarrow} \mathrm{HBrO}_{2}+\mathrm{Ce}(\mathrm{IV}), \\
& \mathrm{HBrO}_{2}+\mathrm{Ce}(\mathrm{IV}) \stackrel{k_{5}}{\rightarrow} \frac{1}{2} \mathrm{HBrO}_{2}, \\
& \mathrm{BrCH}(\mathrm{COOH})_{2}+\mathrm{Ce}(\mathrm{IV}) \stackrel{k_{6}}{\rightarrow} \mathrm{Br}^{-}, \\
& \mathrm{Ce}(\mathrm{IV})+\mathrm{CH}_{2}(\mathrm{COOH})_{2} \longrightarrow \text { inert products. }
\end{aligned}
$$

The concentrations of the principal reactants $\left\{\mathrm{H}^{+}, \mathrm{BrO}_{3}^{-}\right.$, $\left.\mathrm{CH}_{2}(\mathrm{COOH})_{2}\right\}$ in Eqs. (1)-(7) are held constant, leaving four dynamic variables $\left\{\mathrm{Br}^{-}, \mathrm{HBrO}_{2}, \mathrm{Ce}(\mathrm{IV})\right.$, and $\left.\mathrm{BrMA}\right\}$ described by four differential equations. Thus this model explicitly takes into account the dynamics of [BrMA]. The differential equation describing the behavior of $\left[\mathrm{Br}^{-}\right]$is eliminated using the pseudo-steady-state approximation, ${ }^{39}$ leaving three dynamic equations in $\left[\mathrm{HBrO}_{2}\right],[\mathrm{Ce}(\mathrm{IV})]$, and [BrMA], as well as an algebraic expression for $\left[\mathrm{Br}^{-}\right]$given below, in Eq. (13).

\section{THE NONPOLYNOMIAL EQUATIONS}

By introducing the following equivalences:

$$
\begin{array}{rlrl}
X & \equiv\left[\mathrm{HBrO}_{2}\right], & A \equiv\left[\mathrm{BrO}_{3}^{-}\right], \\
Y \equiv\left[\mathrm{Br}^{-}\right], & C \equiv[\mathrm{Ce}(\mathrm{III})]+[\mathrm{Ce}(\mathrm{IV})], \\
Z \equiv[\mathrm{Ce}(\mathrm{IV})], & H \equiv\left[\mathrm{H}^{+}\right], \\
V \equiv[\mathrm{BrMA}], & M \equiv\left[\mathrm{CH}_{2}(\mathrm{COOH})_{2}\right],
\end{array}
$$

as well as the rate constants $k_{1}-k_{7}$ specified in reactions (1)-(7) one obtains the following set of three nonpolynomial 
TABLE I. Numerical values of rate constants and parameters fixed in our simulations, taken from Ref. 20, in the same units.

\begin{tabular}{ll}
\hline \hline$k_{1}=4 \times 10^{6}$ & $k_{2}=2.0$ \\
$k_{3}=3 \times 10^{3}$ & $k_{4}=55.2$ \\
$k_{5}=7 \times 10^{3}$ & $k_{6}=0.09$ \\
$k_{7}=0.23$ & $k_{f}=3.9 \times 10^{-4}$ \\
$A=0.1$ & $C=8.33 \times 10^{-4}$ \\
$H=0.26$ & $M=0.25$ \\
$\alpha=666.7$ & $\beta=0.3478$ \\
\hline \hline
\end{tabular}

differential equations: ${ }^{1,20}$

$$
\begin{aligned}
\frac{d x}{d \tau}= & T_{0}\left\{-k_{1} H Y_{0} x \tilde{y}+k_{2} A H^{2} \frac{Y_{0}}{X_{0}} \tilde{y}-2 k_{3} X_{0} x^{2}\right. \\
& +0.5 k_{4} A^{0.5} H^{1.5} X_{0}^{-0.5}\left(C-Z_{0} z\right) x^{0.5} \\
& \left.-0.5 k_{5} Z_{0} x z-k_{f} x\right\}, \\
\frac{d z}{d \tau}= & T_{0}\left\{k_{4} A^{0.5} H^{1.5} X_{0}^{0.5}\left(\frac{C}{Z_{0}}-z\right) x^{0.5}-k_{5} X_{0} x z\right. \\
& \left.-\alpha k_{6} V_{0} z v-\beta k_{7} M z-k_{f} z\right\}, \\
\frac{d v}{d \tau}= & T_{0}\left\{2 k_{1} H X_{0} \frac{Y_{0}}{V_{0}} x \tilde{y}+k_{2} A H^{2} \frac{Y_{0}}{V_{0}} \tilde{y}+k_{3} \frac{X_{0}^{2}}{V_{0}} x^{2}\right. \\
& \left.-\alpha k_{6} Z_{0} z v-k_{f} v\right\},
\end{aligned}
$$

where

$$
x \equiv \frac{X}{X_{0}}, \quad y \equiv \frac{Y}{Y_{0}}, \quad z \equiv \frac{Z}{Z_{0}}, \quad v \equiv \frac{V}{V_{0}}, \quad \tau \equiv \frac{t}{T_{0}},
$$

with the definitions

$$
\begin{aligned}
& X_{0}=\frac{k_{2}}{k_{5}} A H^{2}, \\
& Y_{0}=4 X_{0}, \\
& Z_{0}=\frac{C A}{40 M}, \\
& V_{0}=4 \frac{A H C}{M^{2}},
\end{aligned}
$$

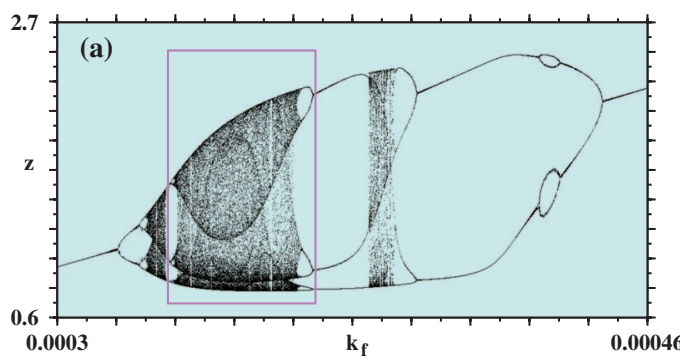

$$
T_{0}=\frac{1}{10 k_{2} A H C} \text {. }
$$

Apart from terms with noninteger exponents, Eqs. (8) and (10) contain an additional nonpolynomial dependence, Eq. (13), arising from elimination of the $\left[\mathrm{Br}^{-}\right]$equation using the pseudosteady state approximation to yield $\tilde{y}$, the scaled, pseudo-steady-state ${ }^{39}$ value of $\left[\mathrm{Br}^{-}\right]$:

$$
\tilde{y}=\frac{\alpha k_{6} Z_{0} V_{0} z v}{\left(k_{1} H X_{0} x+k_{2} A H^{2}+k_{f}\right) Y_{0}} .
$$

The quantities $\alpha$ and $\beta$ are historical artifacts originally defined to separate the reactions of $\mathrm{Ce}(\mathrm{IV})$ with BrMA and $M$; they have no chemical significance. The $\left(C-Z_{0} z\right)$ terms in Eqs. (8) and (9) are inserted intuitively to account for the depletion of $\mathrm{Ce}(\mathrm{III})$ as $\mathrm{Ce}(\mathrm{IV})$ is produced. All parameters appearing in the equations above are collected in Table I, along with the basic numerical values from Ref. 20.

The most important experimental parameter in the BZCSTR system is the inverse residence time, $k_{f}$, (flow rate)/ (reactor volume), which may be very precisely controlled and to which the system is dramatically sensitive. Experiments are typically monitored by measuring $[\mathrm{Ce}(\mathrm{IV})] \equiv Z$ in the CSTR. Thus the independent parameter in nearly all of our calculations is $k_{f}$, and $z$ is sometimes used to demonstrate the dynamic behavior of the model as $k_{f}$ is varied. The other readily controlled experimental parameters are the concentrations of reactants $(A, C, H$, and $M)$ in the feed streams. We assume the constant reactant concentrations in the CSTR are identical to their concentrations in the feed streams.

Figure 1 shows bifurcation diagrams obtained by plotting the local maximal values of $z(t)$ as a function of $k_{f}$. In both diagrams, the $z$ axis was divided into a grid of 600 and the $k_{f}$ axis into 1200 equally spaced values. We integrated Eqs. (8)-(10) using a standard fourth-order Runge-Kutta algorithm with fixed time step $h=2 \times 10^{-6}$. The first $7 \times 10^{4}$ steps were discarded as transient. During the next 140 $\times 10^{4}$ steps we searched for the local maxima of $z$, which were then plotted in the bifurcation diagram. This procedure was repeated for each of the 1200 values of $k_{f}$. Computations

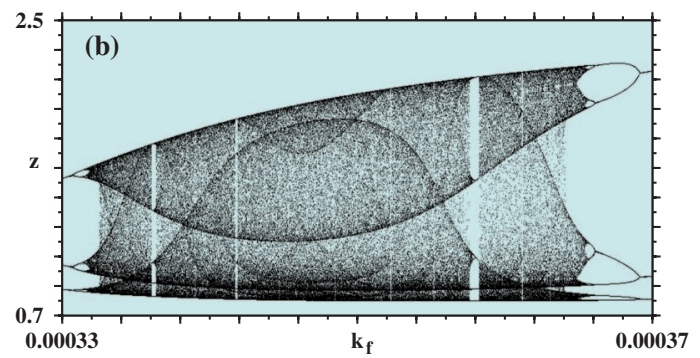

FIG. 1. Bifurcation diagrams illustrating the qualitative similarity of the cascading when either increasing or decreasing the bifurcation parameter. (a) Period-1 solutions exist on both extremes of the diagram. The box marks the region magnified on the right. (b) Period-3 solutions exists on both ends of the diagram. 

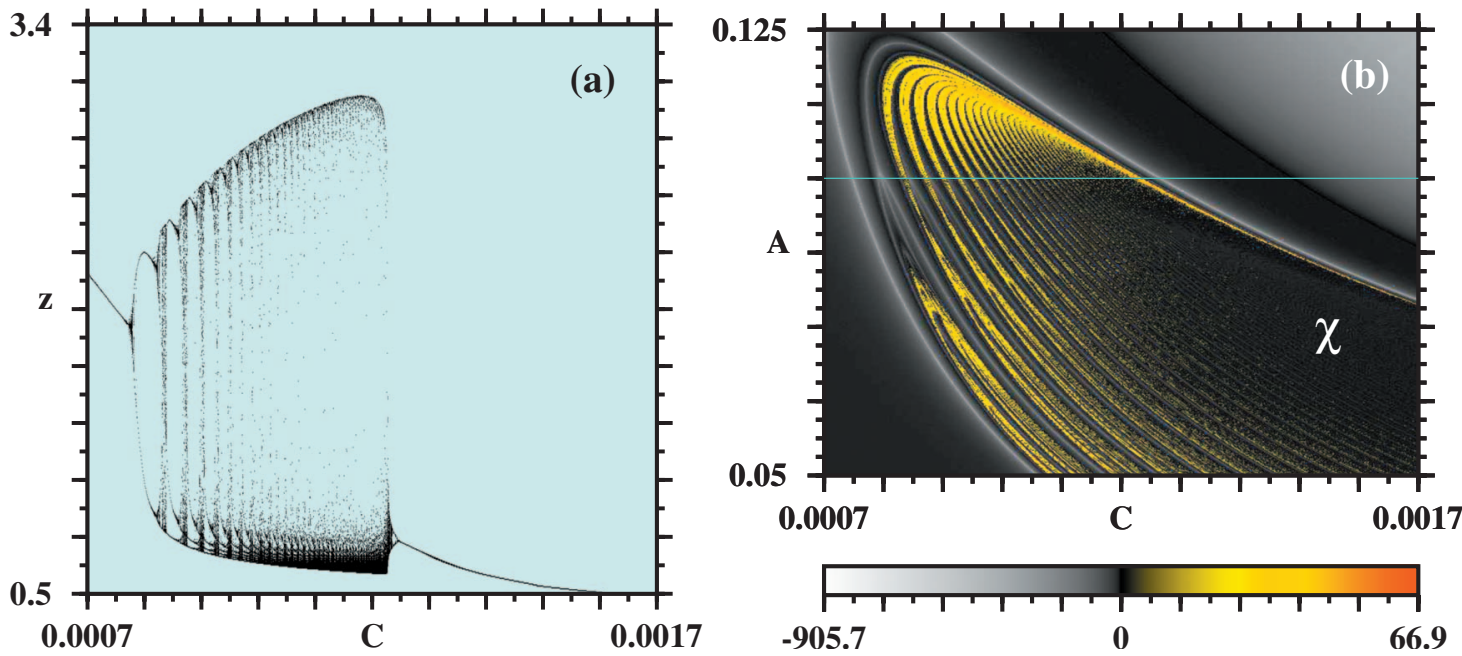

FIG. 2. (a) Bifurcation diagram along $A=0.1$, indicated by the horizontal line in the right panel. (b) Lyapunov phase diagram discriminating periodicity and chaos in the $C \times A$ control space. Colors denote chaos (i.e., positive exponents) while the darker shadings mark periodicity. Chaos prevails in the accumulation region around the letter $\chi$ despite the coloration. The color scale is linear on both sides of zero but not uniform. Note the high spread of exponents, indicating stiffness. The bifurcation diagram has a resolution of $1200 \times 600$ pixels, while the phase diagram displays $600 \times 600$ Lyapunov exponents.

were started at the minimum value of $k_{f}$ from the initial conditions $x_{0}=z_{0}=v_{0}=0.5$ and continued by following the attractor, namely, by using the values of $x, z$, and $v$ obtained at the end of one calculation at a given $k_{f}$ to start a new calculation after incrementing $k_{f}$. This is a standard way of generating bifurcation diagrams, and the rationale behind it is that generically, basins of attraction do not change significantly upon small changes in parameters, thereby ensuring a smooth unfolding of the bifurcation curve.

Comparing the bifurcation diagrams in Fig. 1 above with Fig. 1(a) of Györgyi and Field ${ }^{20}$ one finds that the diagrams are virtually identical, meaning that using Poincaré sections, as done in Ref. 20, or using the maxima of the variable produces identical sequences of bifurcations.

The procedure described above is used to compute all phase diagrams presented in Sec. IV, except that instead of considering local maxima of $z$, all points after the transient were used to calculate the Lyapunov spectrum, i.e., Lyapunov exponents for the three variables of the model. The construction of Lyapunov phase diagrams is a demanding computational task. For instance, to classify a mesh of $N \times N$ parameter points demands the computation of $N^{2}$ basins of attraction, needed to sort out all possible solutions for each parameter set. The computation of each individual basin of attraction requires investigating sets of initial conditions over a $M \times M$ mesh in phase space. The quality of the final diagrams depends sensibly on both $N$ and $M$ being as large as possible. Typically, we used $400 \times 400$ grids, although $600 \times 600$ grids were also used.

\section{PHASE DIAGRAMS}

In this section we present several two-parameter phase diagrams discriminating the dynamic behavior (chaotic or periodic) as $k_{f}$ and one of the reactant concentrations is varied. All parameters are varied over experimentally accessible ranges. Rate constant values are not readily variable experimentally. However, we do present phase diagrams in which the values of $k_{2}$ and $k_{4}$ are varied because these important quantities control important properties of the oscillations and chaos.

Figure 2(a) shows a bifurcation diagram similar to those in Figs. 1(a) and 1(b), but now considering the total ceriumion concentration, $C$, as the bifurcation parameter while keeping $A=0.1$ constant. However what would happen for other values of $A$ close to this one? Figure 2(b) shows how chaos and periodicity are distributed in the $C \times A$ parameter section. It shows that for relatively large variations of $A$ around 0.1 the bifurcation diagram remains essentially the same, apart from an overall stretching.

Figure 2(b) illustrates a typical Lyapunov phase diagram obtained by computing the three Lyapunov exponents for Eqs. (8)-(10), ordering them such that $\lambda_{3}<\lambda_{2}<\lambda_{1}$, and plotting the largest nonzero exponent. We plotted $\lambda_{2}$ whenever $\left|\lambda_{1}\right| \leq 10$. As is well known, ${ }^{40}$ Lyapunov exponents provide a handy quantity allowing one to discriminate between chaos (positive exponents) and periodic oscillation (negative exponents). Figure 2(b) contains a scale of colors that, after suitable renormalization to reflect the extrema of exponents, was used to construct similar figures below where scales are omitted. Noteworthy is the great spread of the magnitude of the exponents, an indication of the stiffness ${ }^{41}$ of the model.

Figure 3 displays phase diagrams for representative parameter sections: $k_{f} \times A, k_{f} \times H$, and $k_{f} \times M$. The top row displays large views of the parameter space, discriminating chaos from periodicity. Two of the diagrams contain the letter $\chi$ to mark, as is done in Fig. 2(b), domains where one finds chaos overwhelmingly, not periodic oscillations as the shadings seem to indicate. To characterize chaos properly, one would need to integrate for much longer times. The middle row shows magnifications of the boxes in the upper row. The thin white spines represent the location of "superstable loci," 12 i.e., loci of local minima of negative Lyapunov exponents. Noteworthy in the middle row are the two rightmost panels displaying fountainlike "eruptions of chaos," a sort of chaos geyser in specific parameter regions in the 

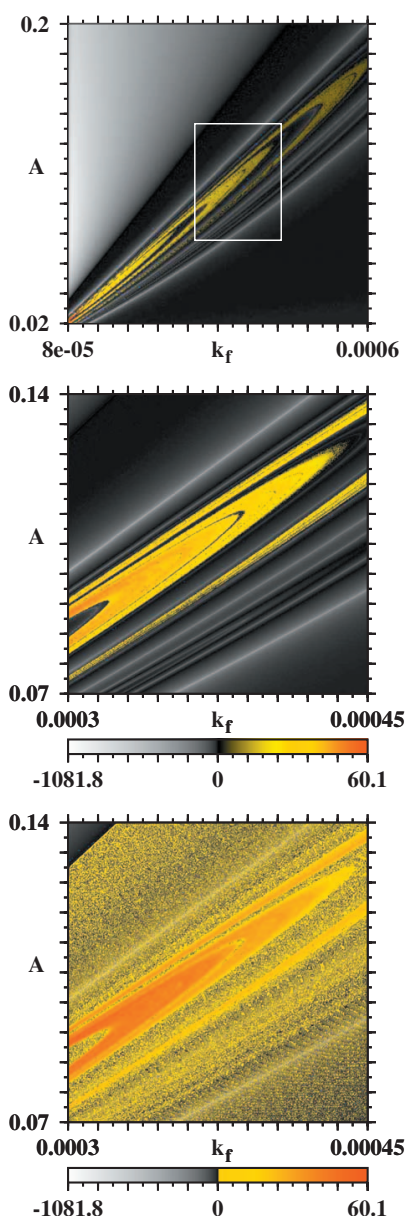
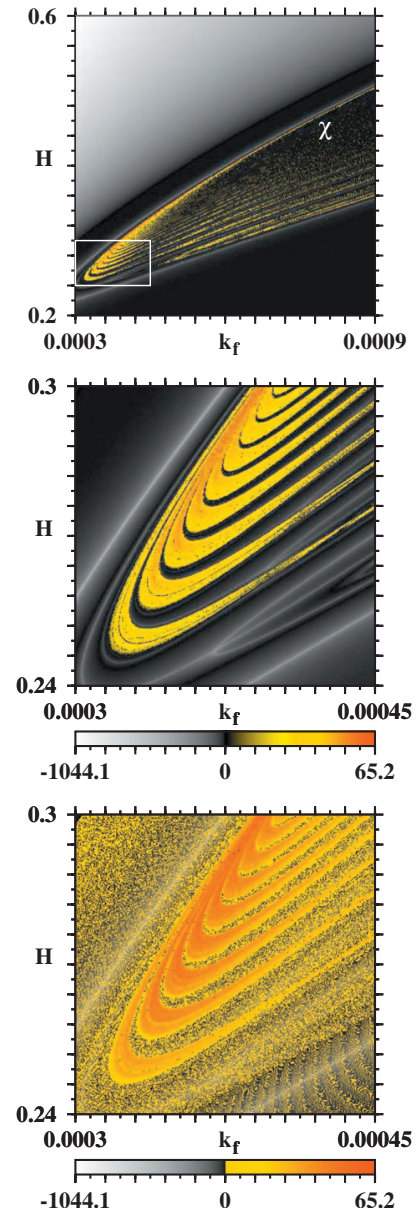
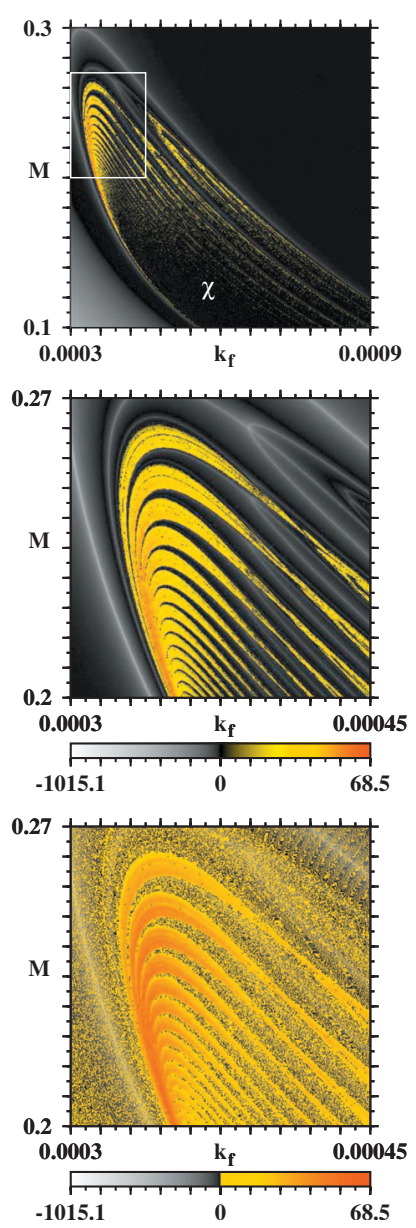

FIG. 3. Top row: phase diagrams illustrating structuring for three distinct parameter cuts over wide parameter ranges. Chaos prevails around the letter $\chi$. Middle row: magnifications of the boxes in the corresponding panel above. Note the great similarity of the two rightmost diagrams with that in Fig. 2(b). Bottom row: replot of the panels in the middle row, but using a strict cut for zero exponents, as indicated by the color scales. To enhance contrast, the intensity of red was slightly increased. The granularity exposes intrinsic difficulties of calculating exponents that are close to zero (see text). $k_{f} \times M$ and $k_{f} \times H$ planes. Eruptions are also present in other parameter sections visible in Fig. 2(b), in the figures below, and in several additional cuts of the parameter space, e.g., $M \times A, M \times H, M \times C$, and $C \times H$ (not shown here).

The bottom row in Fig. 3 illustrates an inherent difficulty of computing Lyapunov exponents: the accurate determination of zero exponents. Away from zero, approximation errors intrinsic to the integration algorithm as well as computational errors occurring in the calculation of exponents remain confined to less significant digits. However, the transition between periodic and chaotic behaviors is marked by zero exponents, where error in the less significant digits is precisely what survives. The bottom row in Fig. 3 is a replot of the panels in the middle row, but using a sharp cut at zero, not the tolerant cut that allows accommodation of numerical uncertainties. In the middle row we plotted $\lambda_{2}$, the second largest exponent, whenever $\lambda_{1}$, the largest exponent, could not be plotted after being "declared" zero, i.e., when it obeyed $\left|\lambda_{1}\right| \leq 10$ (the "tolerance limit"). In the bottom row, we plotted $\lambda_{2}$ whenever $\lambda_{1} \leq 0$ (strict limit). The granularity exposes regions of nearly zero exponents and higher numerical inaccuracies. Even under these very strict plotting requirements, the overall structure of the phase diagrams remains discernible. Recall that Eqs. (8)-(10) are stiff, thus exacerbating numerical difficulties near the line of zero exponents.

Figure 4 shows the relative abundance and organization of chaos and periodicity in the parameter section $k_{f} \times C$, the total cerium-ion concentration. As is clear from the panels in the bottom row, macroscopically, the parameter cuts present two main regimes: a region where chaos and periodicity alternate regularly while experiencing a relatively moderate compression toward each other (exemplified in the right panel), and regions where one finds domains to bend over and strongly accumulate toward well defined limit curves, as seen in the left panel. This structure is surprisingly independent of the parameter explored and markedly distinct from structures recently found for gas and semiconductor lasers, for electric circuits, and for a few other familiar nonlinear oscillators. ${ }^{10,11,15,16}$ In particular, the alternation of chaos and periodicity here is rather different from that found around focal hubs of periodicity. ${ }^{12,16}$

Figure 5 presents progressively more highly resolved phase diagrams obtained when varying simultaneously the rate constants $k_{2}$ and $k_{4}$. The overall organization is similar to that seen above. However in this parameter plane the compression is not as strong as in previous parameter sections, allowing finer details to be recognized even at moderate resolutions. These phase diagrams allow one to understand how the regimes described in Fig. 4 interconnect with each other: by conspicuous "necks," clearly seen in the panels in the upper row, that get less and less pronounced when coming closer and closer to the limit curve, the thick accumulation line dominated by chaos. In the center panel of the middle row one sees period-adding cascades and accumulations similar to those observed in lasers, electric circuits, and 

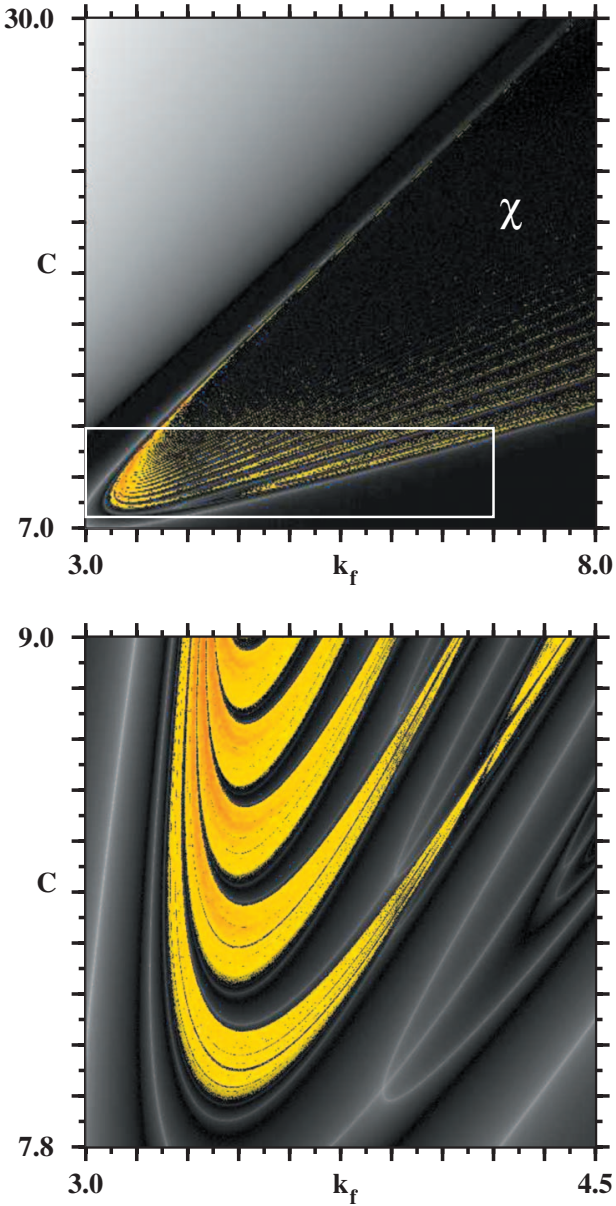
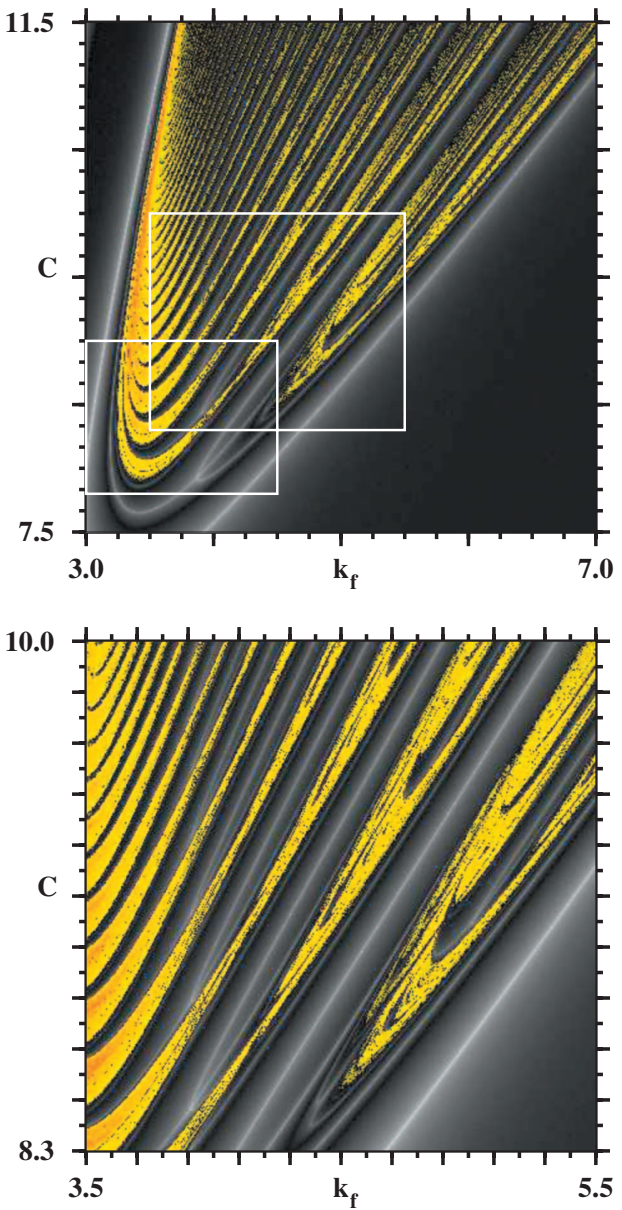

FIG. 4. Successive magnifications illustrating the alternation of periodic and chaotic solutions in the $k_{f} \times C$ space. Note the structural resemblance to parameter cuts shown in Figs. 2(b) and 3. Chaos prevails around the letter $\chi$. Both axis were multiplied by $10^{4}$ to avoid unnecessarily long sequences of zeros. Thus, the minimum values of $k_{f}$ and $C$ in the upper leftmost panel are $3 \times 10^{-4}$ and $7 \times 10^{-4}$, respectively. other nonlinear models. ${ }^{15}$ The bottom row in Fig. 5 illustrates that at higher resolutions, the chaotic domains of the nonpolynomial model display the same recurrent accumulations of shrimps found abundantly in several other models, autonomous or not. ${ }^{6,14-18}$ Shrimps are also found in several additional cuts of the parameter space, e.g., $k_{f} \times M, k_{f} \times H$, and $k_{f} \times C$ (not shown here). It would be interesting to study how periodicity evolves along such accumulations and to quantify their metric properties.

\section{CONCLUSIONS AND OUTLOOK}

This paper reports a detailed numerical investigation of the relative abundance of periodic and chaotic oscillations for a three-variable, 14-parameter, nonpolynomial, autonomous model of the BZR. Although chaotic solutions for BZR models have been known for many years, ${ }^{1-4}$ they were confined to isolated parameter points or to specific onedimensional bifurcation diagrams. No global classification of chaotic phases was attempted. The present work reports phase diagrams discriminating chaos and periodicity along several sections of parameter space. We also describe details of the intertwined structuring of chaos and periodicity over extended experimentally accessible parameter domains.

Globally, on a macroscopic scale, we have shown the structuring of the BZR phase diagrams to display a recurring unusual fountainlike global pattern consisting of eruptions of chaos and periodicities. Such a pattern is very distinct from any structuring based on shrimps and hubs reported recently in literature for prototypical nonlinear oscillators such as gas lasers, semiconductor lasers, electric circuits, or a low-order atmospheric circulation model. The unusual patterns found in the BZR are surprisingly robust and independent of the parameter explored.

Locally, on a microscopic scale, the BZR phase diagrams contain very peculiar hierarchies of parameter networks ending in distinctive and rich accumulation boundaries and structuring similar to that found recently for semiconductor lasers with optical injection, $\mathrm{CO}_{2}$ lasers with feedback, autonomous electric circuits, the Rössler oscillator, Lorenz-84 low-order atmospheric circulation model, and other systems. Since these accumulations of microscopic details were found in all these rather distinct physical models, it seems plausible to expect them to be generic features of flows of codimension two and higher.

In sharp contrast with discrete dynamical systems where periodicities vary always in discrete unitary steps, ${ }^{42}$ chemical reactions provide a natural experimental framework to study how periodicities defined by continuous real numbers evolve and get organized in phase diagrams when several parameters are tuned. Additionally, another enticing open question is whether or not it is possible to infer the presence in phase space of unstable and complicated mathematical phenomena, e.g., homoclinic orbits, based on observation of regularities computed/measured solely in parameter space. Are there clear parameter space signatures of homoclinic orbits? Is it possible to recognize in Lyapunov phase diagrams the loca- 

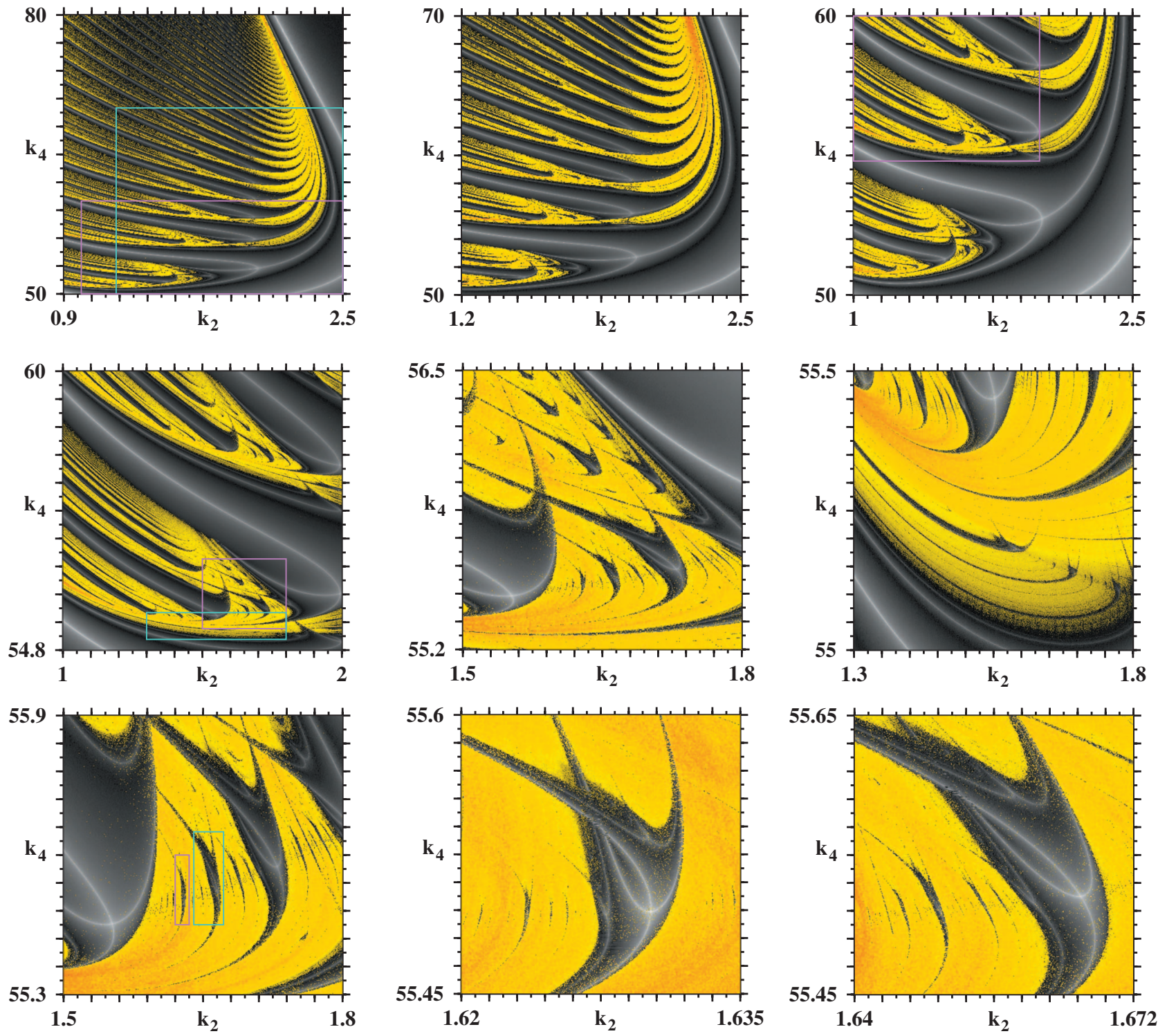

FIG. 5. Progressively more highly resolved phase diagrams illustrating the fine structure of the chaotic phases for selected regions of the $k_{2} \times k_{4}$ parameter space. In the center panel of the middle row one sees period-adding cascades and accumulations similar to those observed in lasers, electric circuits and other models (Ref. 15). The bottom row shows that under high resolution the chaotic phase of the BZR is also riddled with shrimps (Refs. 17 and 18).

tion of parameter loci characterizing simple and multiple (degenerate) homoclinic and heteroclinic phenomena?

Our choice of parameters is motivated and centered around the set originally chosen by Györgyi and Field. ${ }^{20}$ The phase diagrams presented here extend considerably the regimes originally investigated. However, even if using variables reduced by scaling, not the original physical quantities as done here, the effective volume of the parameter space that needs to be explored is huge. Consequently, despite the work reported here, the parameter space still remains mostly unchartered and wanting much more investigation. A sure bet, however, is that journeys through this vast space are bound to reveal interesting dynamics and rich operational points for sustaining individual chemical oscillators and oscillator networks. In particular, they might reveal the mechanism responsible for the novel fountains of chaos which we observed here so frequently and in so many phase diagrams.

\section{ACKNOWLEDGMENTS}

J.G.F. thanks Fundação para a Ciência e Tecnologia, Portugal, for a Posdoctoral Fellowship, and the Instituto de Física da Universidade Federal do Rio Grande do Sul, Porto Alegre, for hospitality. R.J.F. thanks the Department of Chemistry, The University of Montana, for their support. J.A.C.G. thanks Hans J. Herrmann for a fruitful month spent in his Institute in Zürich. He is supported by the Air Force Office of Scientific Research under Grant No. FA9550-07-10102, and by CNPq, Brazil.

${ }^{1}$ R. J. Field and L. Györgyi, Chaos in Chemistry and Biochemistry (World Scientific, Singapore, 1993).

${ }^{2}$ I. R. Epstein and J. A. Pojman, An Introduction to Nonlinear Chemical Dynamics: Oscillations, Waves, Patterns, and Chaos (Oxford University Press, New York, 1998).

${ }^{3}$ P. Gray and S. K. Scott, Chemical Oscillations and Instabilities (Oxford Science, New York, 1990).

${ }^{4}$ S. K. Scott, Chemical Chaos (Oxford Science, New York, 1991).

${ }^{5}$ C. Bonatto, J. C. Garreau, and J. A. C. Gallas, Phys. Rev. Lett. 95, 
143905 (2005).

${ }^{6}$ C. Bonatto and J.A.C. Gallas, Phys. Rev. E 75, 055204(R) (2007).

${ }^{7}$ Y. Zou, M. Thiel, M. C. Romano, J. Kurths, and Q. Bi, Int. J. Bifurcation Chaos Appl. Sci. Eng. 16, 3567 (2006).

${ }^{8}$ V. Castro, M. Monti, W. B. Pardo, J. A. Walkenstein, and E. Rosa, Int. J. Bifurcation Chaos Appl. Sci. Eng. 17, 965 (2007).

${ }^{9}$ H. A. Albuquerque, R. M. Rubinger, and P. C. Rech, Phys. Lett. A 372, 4793 (2008).

${ }^{10}$ G. M. Ramírez-Ávila and J. A. C. Gallas, Revista Boliviana de Fisica 14, 1 (2008).

${ }^{11}$ G.M. Ramírez-Ávila and J.A.C. Gallas, "Quantification of regular and chaotic oscillations of Chua's circuit," IEEE Trans. Circuits-I (submitted).

${ }^{12}$ C. Bonatto and J. A. C. Gallas, Phys. Rev. Lett. 101, 054101 (2008).

${ }^{13}$ J. G. Freire, C. Bonatto, C. DaCamara, and J. A. C. Gallas, Chaos 18, 033121 (2008).

${ }^{14}$ C. Bonatto, J.A.C. Gallas, and Y. Ueda, Phys. Rev. E 77, 026217 (2008).

${ }^{15}$ C. Bonatto and J.A.C. Gallas, Philos. Trans. R. Soc. London, Ser. A 366, 505 (2008).

${ }^{16}$ J. A. C. Gallas, "Infinite networks of periodicity hubs in phase diagrams of simple autonomous flows," Int. J. Bifurcation Chaos Appl. Sci. Eng. (to be published).

${ }^{17}$ J. A. C. Gallas, Phys. Rev. Lett. 70, 2714 (1993); Physica A 202, 196 (1994); Appl. Phys. B: Lasers Opt. 60, S203 (1995); B. R. Hunt, J. A. C. Gallas, C. Grebogi, J. A. Yorke, and H. Koçak, Physica D 129, 35 (1999).

${ }^{18}$ E. N. Lorenz, Physica D 237, 1689 (2008).

${ }^{19} \mathrm{~A}$ phase diagram including the inner structuring of chaotic phases for a nonautonomous chemical flow was reported in Ref. 15.

${ }^{20}$ L. Györgyi and R. J. Field, Nature (London) 355, 808 (1992).
${ }^{21}$ A. P. Nazarea and S. A. Rice, Proc. Natl. Acad. Sci. U.S.A. 68, 2502 (1971).

${ }^{22}$ B. P. Belousov, in Oscillations and Traveling Waves in Chemical Systems, edited by R. J. Field and M. Burger (Wiley, New York, 1985).

${ }^{23}$ A. M. Zhabotinsky, Chaos 1, 379 (1991); Scholarpedia J. 2, 1435 (2007).

${ }^{24}$ R. J. Field, E. Körös, and R. M. Noyes, J. Am. Chem. Soc. 94, 8649 (1972).

${ }^{25}$ R. A. Schmitz, K. R. Graziani, and J. L. Hudson, J. Chem. Phys. 67, 3040 (1977).

${ }^{26}$ J. L. Hudson and J. C. Mankin, J. Chem. Phys. 74, 6171 (1981).

${ }^{27}$ J. S. Turner, J.-C. Roux, W. D. McCormick, and H. L. Swinney, Phys. Lett. A 85, 9 (1981)

${ }^{28}$ C. Vidal, S. Bachelart, and A. Rossi, J. Phys. (Paris) 43, 7 (1982).

${ }^{29}$ J.-C. Roux, Physica D 7, 57 (1983).

${ }^{30}$ L. Györgyi, R. J. Field, Z. Noszticzius, W. D. McCormick, and H. L. Swinney, J. Phys. Chem. 96, 1228 (1992).

${ }^{31}$ L. Györgyi, S. Rempe, and R. J. Field, J. Phys. Chem. 95, 3159 (1991).

${ }^{32}$ I. R. Epstein, Nature (London) 346, 16 (1990).

${ }^{33}$ I. R. Epstein, Nature (London) 374, 321 (1995).

${ }^{34}$ F. W. Schneider and A. F. Münster, J. Phys. Chem. 95, 2130 (1991).

${ }^{35}$ L. Györgyi and R. J. Field, J. Phys. Chem. 92, 7079 (1988).

${ }^{36}$ R. J. Field and R. M. Noyes, J. Chem. Phys. 60, 1877 (1974).

${ }^{37}$ R. J. Field, Scholarpedia J. 2, 1386 (2007).

${ }^{38}$ L. Györgyi and R. J. Field, J. Phys. Chem. 95, 6594 (1991).

${ }^{39}$ R. J. Field, Scholarpedia J. 3, 4051 (2008).

${ }^{40}$ T. Tél and M. Gruiz, Chaotic Dynamics: An Introduction Based on Classical Mechanics (Cambridge University Press, Cambridge, 2006).

${ }^{41}$ J. H. E. Cartwright, Phys. Lett. A 264, 298 (1999).

${ }^{42}$ J. A. C. Gallas, Physica A 283, 17 (2000). 